\title{
Mice with targeted disruption of $p 8$ gene show increased sensitivity to lipopolysaccharide and DNA microarray analysis of livers reveals an aberrant gene expression response
} Sophie Vasseur ${ }^{1}$, Albrecht Hoffmeister ${ }^{1}$, Andrés Garcia-Montero ${ }^{1}$, Marc Barthet ${ }^{1}$, Laure Saint-Michel ${ }^{1}$, Patrice Berthézène ${ }^{1}$, Fritz Fiedler ${ }^{2}$, Daniel Closa ${ }^{3}$, Jean Charles Dagorn ${ }^{1}$ and Juan Lucio Iovanna*1

\author{
Address: ${ }^{1}$ Centre de Recherche INSERM, EMI 0116, 163 Avenue de Luminy, 13009 Marseille, France, ${ }^{2}$ Institut für Anästhesie, Klinikum Mannheim, \\ Theodor-Kutzer-Ufer, D-68167, Mannheim, Germany and ${ }^{3}$ Department of Experimental Pathology. IDIBAPS; IIBB-CSIC, 08036 Barcelona, Spain \\ Email: Sophie Vasseur - vasseur@marseille.inserm.fr; Albrecht Hoffmeister - hofa@medizin.uni-leipzig.de; Andrés Garcia- \\ Montero - angarmon@gugu.usal.es; Marc Barthet - MBARTHET@mail.ap-hm.fr; Laure Saint-Michel - stmichel@inserm-adr.univ-mrs.fr; \\ Patrice Berthézène - berthezene@marseille.inserm.fr; Fritz Fiedler - fritz.fiedler@urz.uni-heidelberg.de; Daniel Closa - dcabam@iibb.csic.es; \\ Jean Charles Dagorn - dagorn@marseille.inserm.fr; Juan Lucio Iovanna* - iovanna@marseille.inserm.fr \\ * Corresponding author
}

Published: 08 September 2003

BMC Gastroenterology 2003, 3:25

This article is available from: http://www.biomedcentral.com/I47I-230X/3/25

(C) 2003 Vasseur et al; licensee BioMed Central Ltd. This is an Open Access article: verbatim copying and redistribution of this article are permitted in all media for any purpose, provided this notice is preserved along with the article's original URL.
Received: 27 March 2003

Accepted: 08 September 2003

\begin{abstract}
Background: $\mathrm{p} 8$ is a DNA-binding protein induced in many tissues in response to LPS treatment. Hence, 88 could be a mediator of LPS-associated effects or, on the contrary, p8 expression may be part of the protective mechanism of the tissues in response to LPS. Finally, p8 expression in response to LPS could also be a simple epiphenomenon.
\end{abstract}

Methods: To investigate the role of $\mathrm{p} 8$ in vivo, we generated p8-deficient mice by gene targeting. Because $p 8$ is a stress protein, we analyzed the response of $p 8^{-/-}$mice to a systemic stress induced by LPS injection. Liver was chosen as model organ to monitor alterations in gene expression.

Results: LPS resulted in higher serum TNF- $\alpha$ concentration and higher mortality rate in p8deficient mice than in wild-type. Also, liver and pancreas, but not lung, from $\mathrm{p}^{-/-}$mice showed increased amounts of MPO and HPO. To gain insight into the molecular bases of such susceptibility, we used high density DNA microarrays consisting of $\sim 6000$ genes and ESTs to compare gene regulation in response to LPS in $\mathrm{p}^{+/+}$and $\mathrm{p} 8^{-/-}$livers. In wild-type, 105 genes and 73 ESTs were upregulated and 232 genes and 138 ESTs down-regulated. By contrast, 212 genes and I 25 ESTs were found up-regulated and 90 genes and 85 ESTs down regulated in $\mathrm{p}^{-1-}$ mice. Among them, only 93 (5I induced and 42 repressed) corresponded to the wild-type pattern, demonstrating that $p 8$ deficiency hinders the normal response to LPS, which may account for the increased sensitivity of p8--mice to the endotoxin.

Conclusions: The large number of genes showing abnormal regulation after LPS suggests that $p 8$ is an important regulatory factor involved in many cellular defence pathways. 


\section{Background}

Using a systematic approach, we identified a new gene, called $p 8$, whose expression is strongly induced during the acute phase of pancreatitis [1]. The deduced protein sequence revealed that $\mathrm{p} 8$ was an 80 aminoacid polypeptide whose primary structure could not be aligned with any of the protein sequences present in public databases. By contrast, p8 contains a canonical bipartite signal for nuclear targeting suggesting that $\mathrm{p} 8$ should be located in the nucleus. Supporting this hypothesis we detected p8 within the nucleus of Cos-7 cells transfected with a p8 expression plasmid, although it was also partly located to the cytoplasm [2]. Furthermore, analysis of p8 primary structure suggested that it should be a DNA-binding protein [1]. More recently, we performed biochemical and biophysical studies showing that human recombinant $\mathrm{p} 8$ was in many structural aspects very similar to the "high mobility group" (HMG) proteins, although sharing with them little aminoacid sequence homology [3]. In addition, p8 binding to DNA, rather weak with the native protein, was strongly enhanced after phosphorylation by PKA of serine/threonine residues [3]. HMG protein binding to DNA is also regulated by post-translational modifications [4]. Thus, in spite of a lack of significant sequence homology, p8 can be considered as a HMG-like protein. It is interesting to note that HMG1, a member of the HMG family of proteins, is a potent pro-inflammatory mediator released passively by necrotic cells but not by apoptotic cells [reviewed in [5]].

Further experiments have shown that p8 activation is not restricted to pancreatic cells. In vivo, p8 mRNA is activated in several tissues in response to systemic lipopolysaccharide (LPS) treatment [6] and in vitro studies showed that a variety of cell lines exhibited transient p8 mRNA expression in response to stress $[1,2]$. p 8 is therefore an ubiquitous protein induced by cellular stress which may be part of a general defence program against cellular injury or cellular stress. Concomitant studies by another laboratory [7] revealed that expression of the candidate of metastasis 1 (Com 1) protein, which is identical to human p8 [2], mediates the growth of tumour cells after metastatic establishment in a secondary organ, suggesting that activated expression of p8 in metastatic cells is required for tumour progression and this hypothesis was recently confirmed [8]. Also, overexpression of p8 in certain cell lines promotes growth $[1,2]$ but it can also inhibit growth when recruited by inhibitory signals [9]. Taken together, these results suggest that $\mathrm{p} 8$ expression may regulate many cellular functions, in response to cellular injury or during tumour development, and that p8 may play different roles depending on the cell type and/or growth conditions.

In response to stress, cells activate various intracytoplasmic pathways, depending on cell type and nature of the stress agent, that ultimately send to the nucleus signals that modulate gene expression. The new pattern of gene expression will help the cells resist the injury and, in case of systemic stress, the efficacy of the defence programs will condition the survival of the organism. Since p8 is activated in several tissues in response to LPS treatment [6], this molecule is a putative mediator of a protective response. To investigate this hypothesis, we have created by targeted disruption mice lacking the $\mathrm{p} 8$ gene. $\mathrm{p} 8^{-/-}$mice showed normal development, they were fertile and their life-span was similar to that of wild type. However, DNA microarray analysis of gene expression in their liver showed important modifications, suggesting that their stress-response capacity could also be altered. Indeed, their mortality rate after LPS challenge was significantly increased compared to wild-type. LPS induced drastic changes in their pattern of gene expression in liver. Interestingly, that pattern was very different from the pattern induced by LPS in wild-type liver, which corresponds to the normal stress response. It was concluded that $\mathrm{p} 8$ is required for adequate response to LPS-induced injury.

\section{Results}

Targeted disruption of the $p 8$ gene creates a null mutation To investigate the function of p8, a null allele was generated in $129 / \mathrm{Sv}$ embryonic stem (ES) cells by replacing exon 2 of the p8 gene, which encodes $60 \%$ of the coding sequence, with a neomycin-resistance cassette. Germline transmission was obtained after injection of these ES cells into C57BL/ 6 blastocysts. Then, we intercrossed mice heterozygous $\left(\mathrm{p}^{+/-}\right)$for the targeted disruption to produce homozygous offspring ( $\left.8^{-/}\right)$. These crosses provided litters of normal size, with living, apparently normal $\mathrm{p} 8 \%$ offspring occurring with a frequency consistent with Mendelian inheritance. p8 deficiency was verified by RT-PCR and/or Southern blot analysis and by immunostaining of the pancreas with acute pancreatitis with a p8 polyclonal antibody (data not shown). Given these results and the extensive nature of the deletion, we concluded that the $\mathrm{p} 8$ mutation was null. Details about production of $\mathrm{p} 8^{-/-}$mice should be obtained from authors upon request.

\section{LPS sensitivity of p8-deficient mice}

p8 was initially defined as a gene activated in several tissues in response to LPS treatment [6]. Hence, p8 could be a mediator of LPS-associated effects or, on the contrary, p8 expression may be part of the protective mechanism of the tissues in response to LPS. p8 expression in response to LPS could also be a simple epiphenomenon. In this study, we analyzed whether p8 knockout mice would display altered reactivity to LPS. Groups of 20 mice each were injected with $70 \mathrm{mg} / \mathrm{kg}$ and lethality was monitored. As shown in Figure 1,70\% of p8\% mice died 36 hours after LPS treatment, compared to only $20 \%$ of $\mathrm{p} 8^{+/+}$mice. After

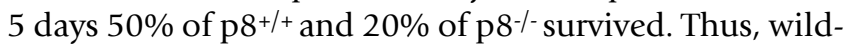




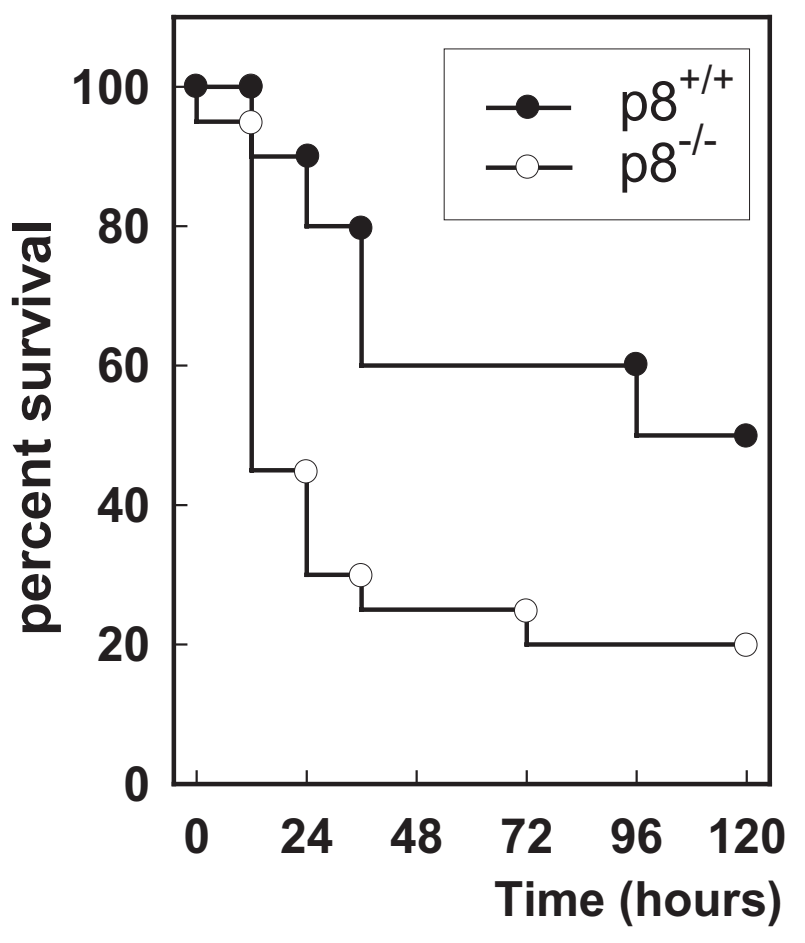

Figure I

Lipopolysaccharide (LPS)-induced mortality in 129/Sv wildtype and 129/Sv-backcrossed p8-deficient knockout mice. Groups $(n=20)$ of female mice were injected intraperitoneally with $70 \mathrm{mg} / \mathrm{kg}$ LPS (Salmonella thyphosa), and survival was monitored every 12 hours for 5 days.

type mice tolerated LPS treatment better than $\mathrm{p} 8 \%$ mice. We monitored in parallel the serum levels of three cytokines. If levels of interferon $\gamma$ (IFN $\gamma$ ) and the antiinflammatory cytokine IL-10 were not different in $\mathrm{p}^{-/}$ and $\mathrm{p}^{+/+}$mice (data not shown), the level of TNF- $\alpha$ was significantly higher in $\mathrm{p} 8 \%$ mice (Figure 2 ), supporting the hypothesis that $\mathrm{p} 8$ expression provides some protection against LPS aggression. Finally, whether liver or pancreas could be differently affected by LPS challenge depending on the p8 status was evaluated by monitoring serum levels of GOT and amylase. No difference was observed (data not shown). Although these markers are not very sensitive, these results suggest that, in these two organs, lack of p8 expression does not increase markedly LPS-induced cell damage.

\section{Myeloperoxidase (MPO) and hydroperoxide (HPO) in lung, liver and pancreas after LPS treatment}

LPS is known to induce a systemic inflammatory syndrome characterized by strong leucocytic infiltration and free radical production which may be lethal [10]. To

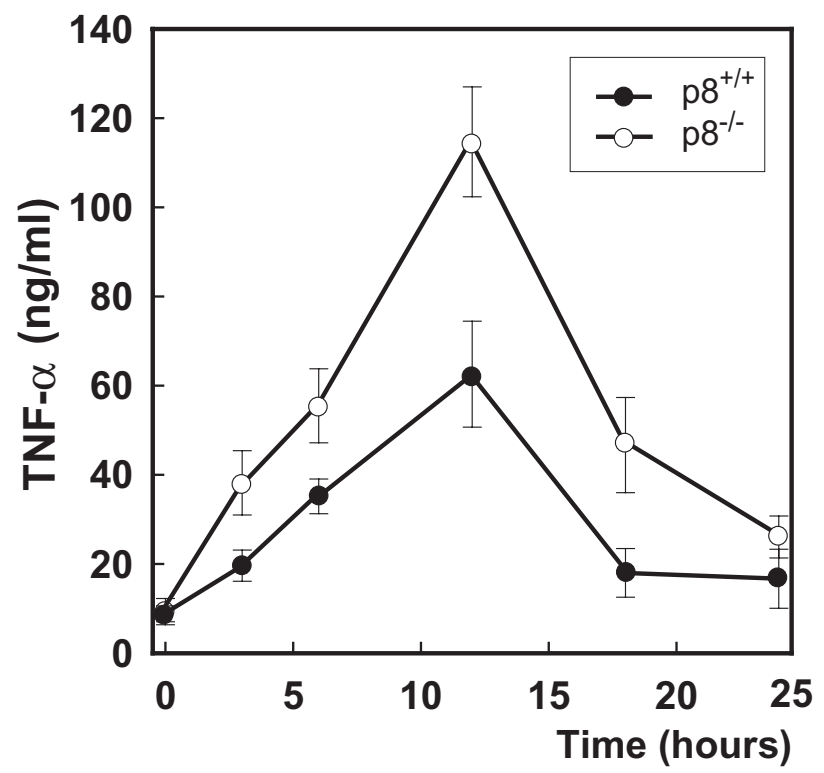

Figure 2

Wild-type and p8-deficient mice were injected intraperitoneally with $70 \mathrm{mg} / \mathrm{kg}$ LPS (Salmonella thyphosa), and serum samples were harvested after diferent times. TNF- $\alpha$ levels were determined by ELISA. Errors bars represent the standard deviation $(n=6)$.

investigate the role of $\mathrm{p} 8$ expression during LPS treatment we monitored MPO activity, as an indicator of tissue infiltration, and HPO concentration as an indicator of free radical production, known to correlate with the intensity of lesions. In control mice, liver, lung and pancreas showed increased MPO activity and HPO concentration after LPS injection, as expected (Figures 3, 4 and 5). In p8I- mouse lungs, the kinetics of changes in MPO activity and HPO were the same as in wild-type mice (Figure 3). They were however very different in pancreas and liver, showing that $\mathrm{p} 8$ influence could vary among tissues. In liver from control mice, MPO activity increased sharply to a maximum already reached 6 hours after LPS injection, then dropped by $65 \%$ in the next 6 hours and decreased more progressively thereafter (Figure 4 ), indicating that liver recruited leucocytes for transient infiltration. In liver from p8-deficient mice, MPO activity increases progressively with a maximum at 18 hours suggesting that the mechanism of leucocyte recruitment was either altered or different from wild-type. Contrary to MPO activity, HPO production was similar in $\mathrm{p}^{+/+}$and $\mathrm{p} 8 \%$ mouse livers. This is not surprising since HPO is produced by Kupffer cells [11] which are specialized macrophages residing in the liver. In pancreas from control mice, changes in MPO 

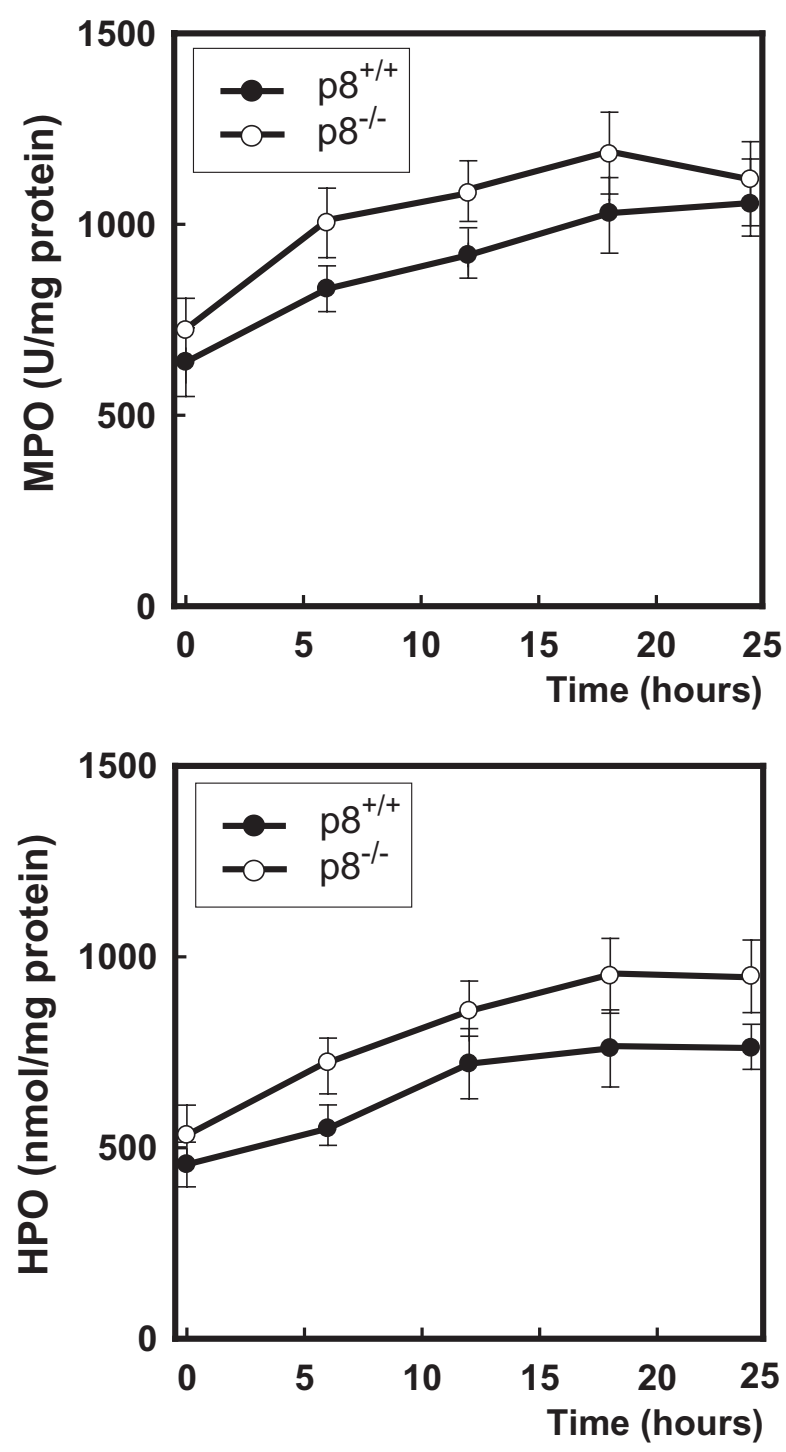

Figure 3

Wild-type and p8-deficient mice were injected intraperitoneally with $70 \mathrm{mg} / \mathrm{kg}$ LPS (Salmonella thyphosa), and Myeloperoxidase (MPO) activity and Hydroperoxide (HPO) concentration were measured in lung after $6,12,18$ and 24 hours. Errors bars represent the standard deviation $(n=7)$.

and HPO followed the same pattern, i.e. transient increase with maximum 12 hours after LPS injection followed by decrease towards control values. In p8-deficient mice, both MPO activity and HPO concentration remained elevated after 12 hours (Figure 5) underscoring the role of $\mathrm{p} 8$ in the regulation of infiltration and free radical production. Taken together, these results suggest that $\mathrm{p} 8$ contrib-
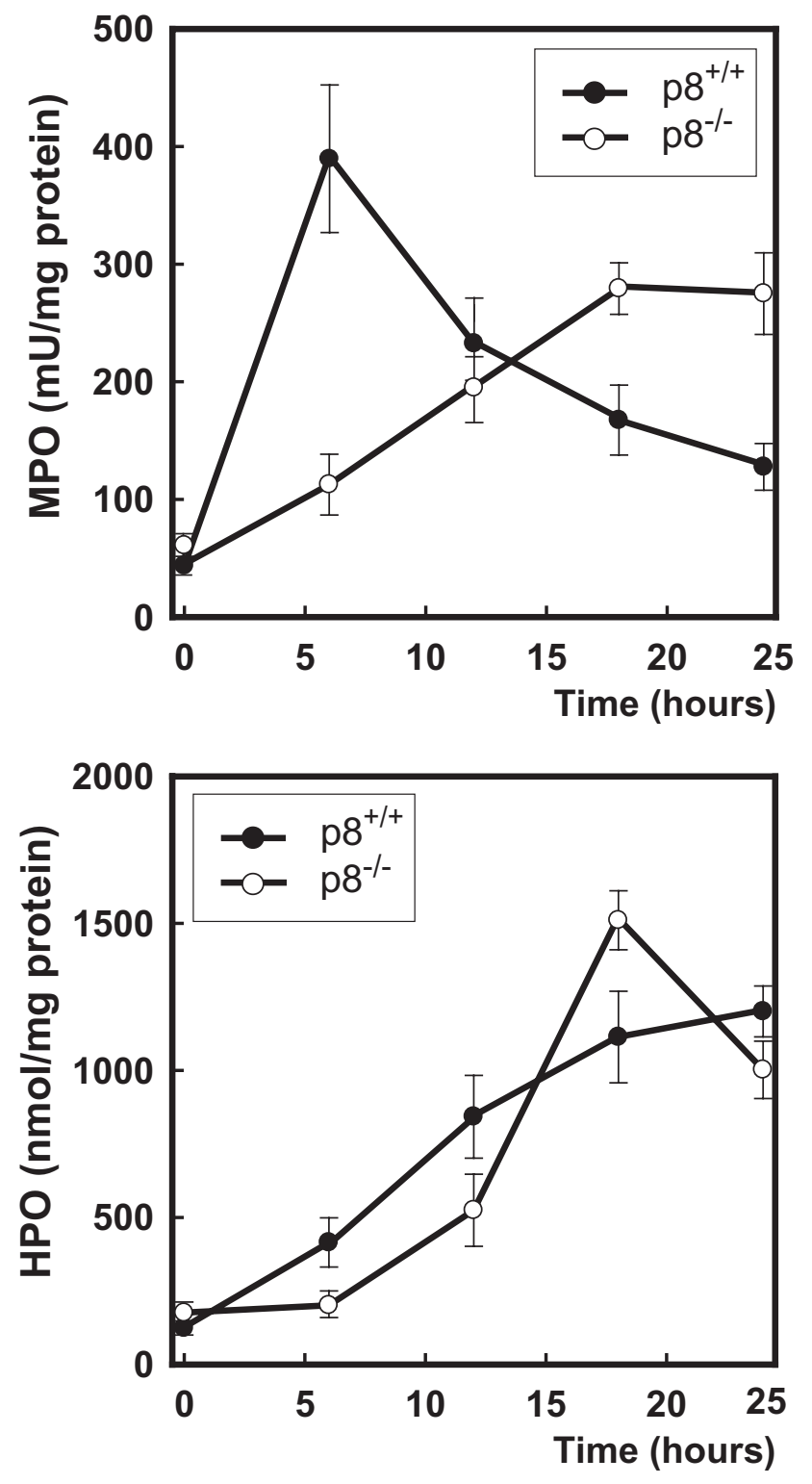

Figure 4

Wild-type and p8-deficient mice were injected intraperitoneally with $70 \mathrm{mg} / \mathrm{kg}$ LPS (Salmonella thyphosa), and Myeloperoxidase (MPO) activity and Hydroperoxide (HPO) concentration were measured in liver after $6,12,18$ and 24 hours. Errors bars represent the standard deviation $(n=7)$.

utes to the tissue response to LPS, such response being different in pancreas, liver and lung.

\section{Gene expression in liver analyzed by DNA microarray}

Liver was chosen as a target tissue of the LPS to decipher the role $\mathrm{p} 8$ in constitutive gene expression and in the response to LPS. We compared the expressions of $\sim 6,000$ 

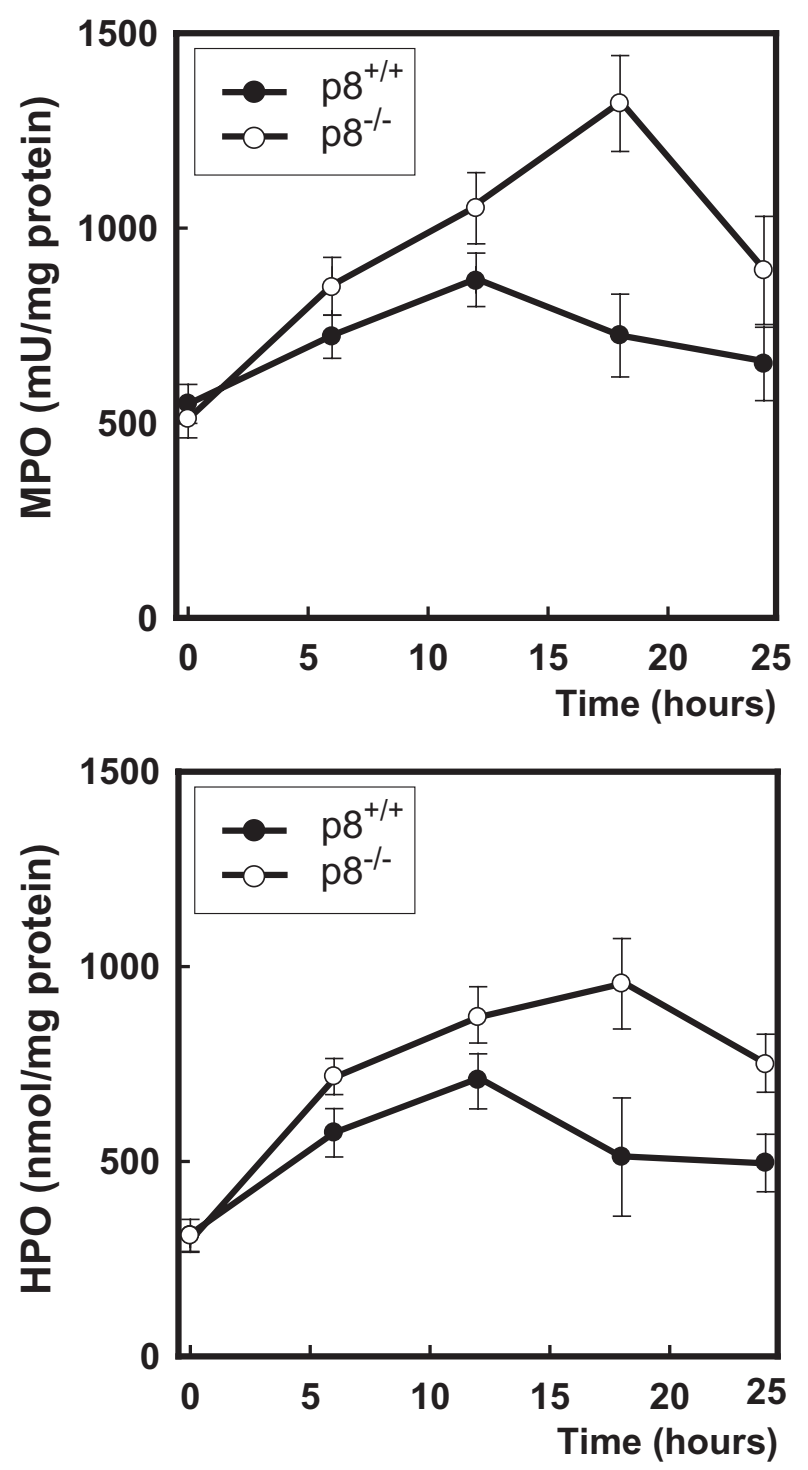

\section{Figure 5}

Wild-type and p8-deficient mice were injected intraperitoneally with $70 \mathrm{mg} / \mathrm{kg}$ LPS (Salmonella thyphosa), and Myeloperoxidase (MPO) activity and Hydroperoxide (HPO) concentration were measured in pancreas after $6,12,18$ and 24 hours. Errors bars represent the standard deviation $(\mathrm{n}=$ 7).

different genes in liver from $\mathrm{p}^{+/+}$and $\mathrm{p} 8^{-/-}$mice, without treatment and 12 hours after an intraperitoneal injection of LPS.

\section{Gene expression in livers from $\mathrm{p}^{+/+}$and $p 8^{-/-}$mice}

The gene expression pattern of $\mathrm{p} 8^{-/-}$mouse liver was compared to that of wild-type, in basal conditions, using DNA microarray analysis. Only genes whose expression differed by a factor $\geq 2$ were considered (Tables 1 and 2 [see Additional file 1]). Of the 6,000 known genes and ESTs monitored, 156 (2.6\%) showed consistent differences. As control, expression of several genes chosen at random was measured by RT-PCR analysis and results confirmed microarray data (not shown). In $\mathrm{p} 8 \%$ liver, the expression of 78 known genes and 33 ESTs (1.85\%) was lowered (by a factor of 2 to 8 ), and that of 25 known genes and 20 ESTs $(0.75 \%)$ was increased (by a factor of 2 to 11.1$)$ compared to wild-type. Of the 78 known genes down-regulated as a result of p8 deficiency, 14 were implicated in protein synthesis, transport or post-translation modifications, 11 were implicated in signaling, 7 coded for secretory factors and 2 for cytoskeletal proteins, 13 were transcription factors or involved in RNA processing, 3 coded for apoptosisrelated proteins, 7 corresponded to membrane or transporter proteins, 17 to cytosolic enzymes or detoxification factors, and 4 were proteins implicated in cell growth. Among genes up-regulated as a consequence of p8 deficiency, 6 corresponded to signaling molecules, 4 coded for secretory factors, 3 for cytoskeletal proteins, 5 for transcription factors or for proteins involved in RNA processing, 1 was apoptosis-related, 4 corresponded to membrane and transporter proteins and 2 were cytosolic enzymes. The list of ESTs up- or down-regulated by a factor of at least 2 is available at http://www.mar seille.inserm.fr/Site INSERM/emi0116/resultsESTs.htm.

\section{Gene expression in liver after LPS treatment}

Pure SV129J mice were treated with $70 \mathrm{mg} / \mathrm{kg}$ of LPS from Salmonella thyphosa and gene expression in the liver was compared to expression in the liver of mice injected with saline. Expression of 105 known genes (1.77\%) and 73 ESTs (1.22\%) was up-regulated (by a factor of 2 to 62.3 ) whereas expression of 232 (3.87\%) known genes and 138 ESTs $(2.30 \%)$ was down-regulated (by a factor from 2 to 68.3). Known genes up-regulated by LPS treatment were grouped as follows: 4 genes coded for proteins implicated in protein synthesis, transport or post-translational modifications, 17 for proteins involved in signaling, 23 genes corresponded to secretory factors, 5 to cytoskeletal proteins, 27 to transcription factors or molecules involved in RNA processing, 1 gene coded for an apoptosis-related protein, 19 corresponded to membrane or transporter proteins, 5 to cytosolic enzymes and detoxification factors, and 4 were implicated in cell growth. Among known genes down-regulated as consequence of LPS treatment, 23 were implicated in protein synthesis, transport or posttranslational modifications, 34 corresponded to signaling molecules, 16 were secretory factors, 7 coded for cytoskeletal proteins, 20 for transcription factors and RNA processing proteins, 5 coded for apoptosis-related proteins, 31 corresponded to membrane or transporter proteins, 79 to cytosolic enzymes or detoxification factors, 
and 17 corresponded to growth-related factors. Results are shown in Tables 3 and 4 [see Additional file 2]. The list of ESTs up- or down-regulated by LPS by a factor of at least 2 is available at http://www.marseille.inserm.fr/ Site INSERM/emi0116/resultsESTs.htm.

\section{Gene expression in p $^{\text {-l- }}$ - liver after LPS treatment}

Effect of LPS treatment on liver gene expression was also analyzed in $\mathrm{p}^{-\%}$ mice. Results are presented in Tables 5 and 6 [see Additional file 3]. Five hundred and twelve $(8.53 \%)$ showed consistent changes in expression level of at least 2-fold. Expression of 212 known genes and 125 ESTs (2.08\%) was up-regulated (by a factor of 2 to 50.0), and 90 known genes and 85 ESTs (1.42\%) were down-regulated (by a factor of 2 to 29.9). Among up-regulated genes, 30 coded for proteins implicated in protein synthesis, transport or post-translation modifications, 45 for genes involved in signaling, 22 corresponded to secretory factors, 16 to cytoskeletal proteins, 34 to transcription factors and RNA processing molecules, 7 genes encoded apoptosis-related proteins, 34 corresponded to membrane or transporter proteins, 13 to cytosolic enzymes or detoxification factors, and 11 to proteins implicated in cell growth. Among down-regulated genes, 1 coded for a mitochondrial stress protein, 8 corresponded to signaling molecules, 10 to secretory factors, 3 to cytoskeletal proteins, 9 encoded transcription factors or proteins involved in RNA processing, 1 to an apoptosis-related protein, 10 corresponded to membrane or transporter proteins, 45 to cytosolic enzymes and detoxification factors, and 3 corresponded to growth-related factors. To be physiologically relevant, modifications in gene expression observed in $\mathrm{p} 8 \%$ liver after LPS have to be significant compared to normal wild-type liver (e.g.: a gene down-regulated by a factor of 2 in $\mathrm{p}^{-\%}$, compared to wild-type, must be up-regulated at least 4 times after LPS to be physiologically significant). Interestingly, it was indeed the case for more than $90 \%$ of genes up-regulated and $99 \%$ of genes down regulated after LPS. ESTs up- or down-regulated by LPS in p $8 \%$ liver by a factor of at least 2 are available at http:www.marseille.inserm.fr/Site INSERM/emi0116/ result sESTs.htm.

\section{Comparison of LPS-induced gene regulations in $p^{8+/+}$ and $p^{8-1-}$ livers}

If the total number of genes with significantly altered expression after LPS was similar in $\mathrm{p}^{+/+}$and $\mathrm{p} 8^{-/-}$livers, the regulatory patterns were very different. Twice more genes were upregulated in $\mathrm{p}^{-/}$than in $\mathrm{p}^{+/+}$mice (337 vs. $178)$, the proportion being reversed for down-regulated genes (175 vs. 370). Among known genes, only 51 and 42 were up- and down-regulated, respectively, in both $\mathrm{p} 8^{+/+}$ and $\mathrm{p} 8 \%$ livers. More strikingly, several genes were oppositely regulated, including 19 genes down-regulated in $\mathrm{p}^{+/+}$but up-regulated in $\mathrm{p}^{-/-}$liver and one gene up-regu-

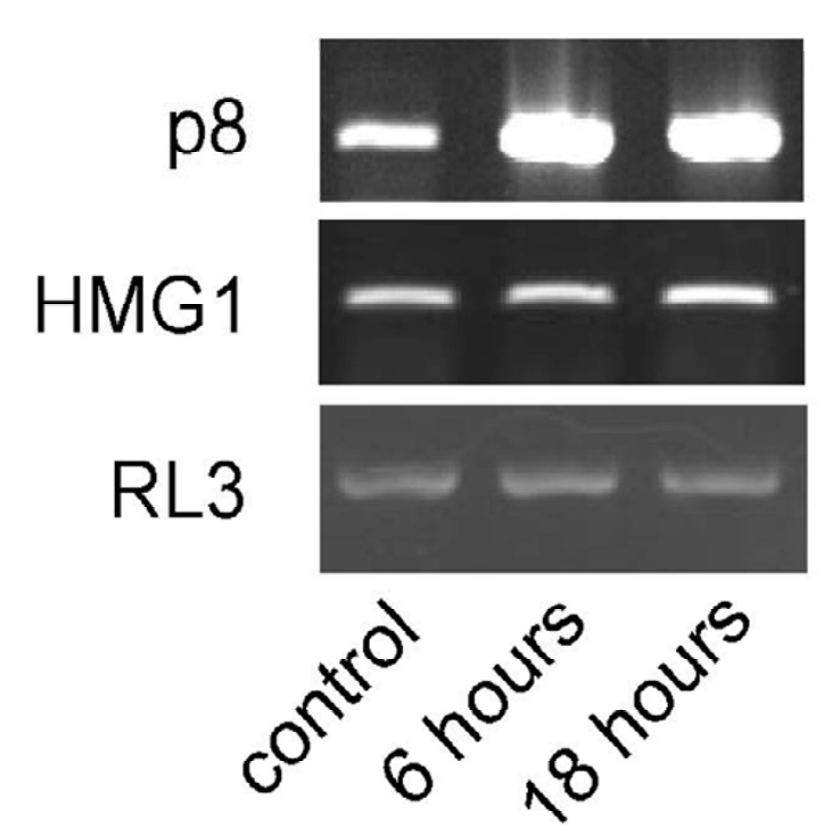

Figure 6

Wild-type mice were injected intraperitoneally with $70 \mathrm{mg} /$ kg LPS (Salmonella thyphosa), and mRNA expression was measured in liver after 6 and 18 hours. RNA was extracted using Trizol procedures and I $\mu \mathrm{g}$ RNA was analyzed by RTPCR using specific primers for HMG I, p8 and ribosomal protein L3 (RL3) as a control as described in Material and Methods.

lated in $\mathrm{p}^{+/+}$but down-regulated in $\mathrm{p} 8^{\%-}$ liver, which underscores the differences between LPS-induced gene regulation patterns in $\mathrm{p}^{+/+}$and $\mathrm{p} 8^{-/}$livers.

\section{HMGI expression in liver after LPS treatment}

Finally, because the effects of HMG1 and p8 seem to be opposite (e.g.: HMG1 play a pro-inflammatory role whereas $\mathrm{p} 8$ acts as an anti-inflammatory factor), we analyzed in detail expression of HMG1 mRNA by semiquantitative RT-PCR. Contrary to the HMG-related proteins HMG-I(Y) and $\mathrm{p} 8$ which are activated in liver by LPS treatment, HMG1 mRNA expression remains unchanged after 6 and 18 hours (Figure 6). This is in fact not a surprise since HMG1 expression in liver by LPS seems to be activated by $2-3$ folds within the first 2 hours following LPS treatment, returning to the untreated value thereafter [12]. Another important difference to be noted is that whereas $\mathrm{p} 8 \%$ mice were normal, $\mathrm{HMG} 1 \%$ pups are born alive, but die within 24 hours due to hypoglycemia [13]. 


\section{Discussion}

We constructed a p8\%-mouse as a tool to investigate the function of the p8 gene. p8-deficient mice were fertile, developed normally and showed no obvious signs of disease up to 20 months of age. These findings imply either that $\mathrm{p} 8$ is not involved in crucial developmental processes or that other genes are functionally redundant. However, p8-deficient mice were more sensitive to the noxious effects of LPS, as evidenced by decreased survival (Figure 1), increased levels of serum TNF- $\alpha$ (Figure 2) and higher levels of MPO and HPO in some tissues (Figure 3, 4 and 5), compared to wild-type, which suggests the implication of p8 in important defence mechanisms. Because $\mathrm{p} 8$ is biochemically related to HMG-I(Y) proteins [3], which play a critical role in architectural changes of DNA to modulate gene expression [14-17], we made the hypothesis that, under conditions of stress, p8 regulates gene expression to improve cell resistance. Lack of p8 expression would therefore prevent adequate stress response with, as consequence, increased mouse mortality. Liver was chosen as model organ to test that hypothesis, because it is known that LPS triggers in that organ important changes in the expression of several genes $[18,19]$. To analyze in $\mathrm{p} 8 \%$ mice how the lack of $\mathrm{p} 8$ might alter liver response to LPS, we documented by microarray DNA studies the pattern of gene expression in p8\%- liver, for comparison with wild-type, then the response of wild-type liver to LPS for eventual comparison with $\mathrm{p} 8 \%$ - liver.

\section{Gene expression is altered in p $^{-/-}$liver}

As speculated, the pattern of liver gene expression was strongly altered as a consequence of $\mathrm{p} 8$ gene disruption. Compared to wild-type, 156 genes (2.6\%) showed significant changes in their expression (111 repressed and 45 induced). Therefore, many genes are either directly dependent on $\mathrm{p} 8$ for adequate expression, or indirectly modulated in response to perturbations resulting from its absence. p8 seems to be preferentially, but not exclusively, a positive regulator since more than two thirds of the genes were down-regulated in the $\mathrm{p} 8$-/- liver. Interestingly, p8 interferes with most cellular functions since genes altered upon p8 disruption were involved in transcription and RNA processing, protein synthesis, transport and post-translational modification, apoptosis, regulation of cell growth, intracellular signaling, or encoded secretory or cytoskeleton proteins, membrane and transporter proteins, cytosolic enzymes and detoxification factors.

\section{Liver response to LPS is altered in $\mathbf{p 8}^{-/-}$mice}

Modulation by LPS of liver gene expression was first assessed in wild-type mice. Among the 6000 known genes and ESTs of the microarray, 548 (9.1\%) showed significant change (at least twofold) in their expression. Upregulation was observed for 178 genes, whereas 370 genes were down-regulated. As shown in Tables 3 and 4 [see Additional file 2], genes implicated in all major intracellular pathways are involved in the liver response to LPS. Such a wide spectrum of changes after endotoxin treatment was already reported in other tissues [20-22]. Although the influence of LPS on liver is not the main objective of this work, it is noteworthy that many interesting genes are found strongly up-regulated (e.g.: proteasome subunits, PAI-I, MIG, mag-1, Scya 5) or downregulated (EGF receptor-binding protein, MHL-1, IL-6 receptor), underscoring that LPS had triggered a very elaborate response.

In $\mathrm{p} 8 \%$ mice, liver gene expression was also strongly modified by LPS. To our surprise, the total number of genes with altered expression was almost the same as in wild type (512 vs. 548). However, only 93 of them (1.55\%), because they show the same regulation (up- or down) as in wild-type, would be regulated after LPS challenge through p8-independent pathways. Therefore, a large number of genes whose regulation is part of the normal response to LPS could not be properly modulated in $\mathrm{p} 8 \%$ mice. Because these genes are involved in major cellular functions (Tables 3, 4, 5 and 6 [see Additional files 2 and $3)$, such alteration could be partly responsible for the increased sensitivity of $\mathrm{p} 8 \%$ mice to LPS, inasmuch as other organs should be similarly affected. Some genes previously reported as protective against LPS challenge, such as GARG-16 [23], SAA5 [24] and SOD3 [25] were actually found overexpressed after LPS in wild-type but not in $\mathrm{p} 8 \%$ - livers. Other genes identified in this systematic study could therefore lead to novel therapeutic strategies in endotoxin-mediated pathologies.

In addition to preventing adequate regulation of many genes involved in the normal response to LPS, lack of p8 perturbated the expression of a large number of other genes, which were improperly up- or down-regulated. These genes belonging to many cellular pathways, further intracellular perturbations are expected which may amplify the noxious consequences of LPS administration. Among genes specifically activated by LPS in p8-deficient liver, the chromatin nonhistone high mobility group protein HMG-I(Y) gene (accession \# J04179) was of particular interest because the corresponding proteins are structurally and biochemically similar to p8, which suggests similar functions [[3] and unpublished results]. In response to LPS, expression of $\mathrm{p} 8$ is strongly overexpressed in liver [6] whereas HMG proteins are not activated (Table 3 [see Additional file 2]). Yet, in p8-deficient mouse liver, the HMG-I(Y) protein was activated 8.5 fold, which may reflect a compensatory mechanism. These results strongly suggest that $\mathrm{p} 8$, which is probably a transcription factor [26], is a key regulatory gene in the cellular response to LPS. If its absence is apparently without 
important consequences in normal conditions, it prevents mice from fully developing their defence program against LPS challenge.

In conclusion, this work demonstrates that $\mathrm{p} 8$ overexpressed in response to LPS is not a mediator of tissue insult but rather an important component of a defence program. Microarray DNA analysis of liver gene expression demonstrated the crucial role of the protein in the regulation of important cellular pathways. If the technical limitation of that analysis to roughly one fifth of the genome prevented thorough description of the $\mathrm{p} 8$ pathway, the information gathered should provide ground for many further studies concerning p8 function and the cellular response to endotoxin challenge.

\section{Methods}

\section{Targeted disruption of the mouse $p 8$ gene}

The construction of the targeting vector used in the targeted disruption of the $\mathrm{p} 8$ locus in embryonic stem cells of mouse strain $129 / \mathrm{Sv}$ was previously reported by Kuhbandner et al. [27]. ES cells from three heterozygous clones for the mutated p8 allele were injected into blastocysts of C57BL/6 mice. We obtained in the littermates 7 male mice highly chimerical. They were intercrossed with wild-type C57BL/ 6 females and 4 showed germline transmission. Genotype identification was done by PCR and/ or Southern blot with genomic DNA prepared from tail biopsies of 10 day-old mice. Heterozygous mice for the mutated p8 allele were obtained and used in breeding experiments to generate mice homozygous $(-/)$ for the disruption of p8. p8-deficient mice were backcrossed to SV129J mice for nine generations as recently described [28].

\section{Lipopolysaccharide toxicity in vivo}

Mice were housed in rooms maintained at constant temperature and humidity and subjected to a 12 -h light/dark cycle. Mice received normal rodent chow (Purina, St. Louis, MO) and water ad libitum. Three months old females were used. Animals ( $\mathrm{p} 8^{+/+}$and $\mathrm{p} 8^{-/}$) were injected intraperitoneally with $70 \mathrm{mg} / \mathrm{kg}$ of LPS (Salmonella thyphosa, Sigma) resuspended in sterile saline. This dose of LPS was chosen on the basis of preliminary studies in wild-type $129 / \mathrm{Sv}$ mice in which the dose resulted in less than $50 \%$ mortality. Deaths were recorded every 12 hours, and mice that survived 5 days had fully recovered and were considered as long-term survivors.

\section{Serum GOT and amylase measurements}

Amylase and GOT activities were determined with commercially available assays kits from Roche Biochemicals and Sigma Diagnostics respectively following manufacturer recommendations.

\section{Determination of TNF- $\alpha$}

For determination of TNF- $\alpha$ release kinetics in serum, mice were killed at the indicated time points after treatment, and serum samples were harvested. TNF- $\alpha$ serum level was determined with a commercially available enzyme-linked immunoabsorbent assay (ELISA) kit (R\&D Systems).

\section{Myeloperoxidase assay}

Myeloperoxidase was measured photometrically with $3,3^{\prime}, 5,5^{\prime}$-tetramethylbenzidine as substrate as previously described [29]. Samples were macerated with $0.5 \%$ hexadecyltrimethylammonium bromide in $50 \mathrm{mM}$ phosphate buffer $\mathrm{pH}$ 6.0. Homogenates where then disrupted for 30 s using a Labsonic (B Braun) sonicator at $20 \%$ power and submitted to three cycles of snap freezing in dry ice and thawing before a final $30 \mathrm{~s}$ sonication. Samples were incubated at $60^{\circ} \mathrm{C}$ for 2 hours and then spun down at $4000 \times$ $g$ for $12 \mathrm{~min}$. Supernatants were collected for myeloperoxidase assay. Enzyme activity was assessed photometrically at $630 \mathrm{~nm}$. The assay mixture consisted of $20 \mu \mathrm{l}$ supernatant, $10 \mu \mathrm{l}$ tetramethylbenzidine (final concentration $1.6 \mathrm{mM}$ ) dissolved in $\mathrm{DMSO}$, and $70 \mu \mathrm{l} \mathrm{H}_{2} \mathrm{O}_{2}$ (final concentration $3.0 \mathrm{mM}$ ) diluted in $80 \mathrm{mM}$ phosphate buffer pH 5.4.

\section{Hydroperoxide assay}

Tissue samples were homogenized and lipid hydroperoxides were extracted with a mixture of sample:Methanol:Chloroform 1:1:2. The chloroform extract was carefully collected and used to measure the hydroperoxide concentration with a commercially available assay kit from Cayman Chemical (Ann Arbor, MI).

\section{High density oligonucleotide microarrays}

Mice $\mathrm{p}^{+/+}$and $\mathrm{p} 8 \%$ were injected with $70 \mathrm{mg} / \mathrm{kg}$ of LPS (Salmonella thyphosa, Sigma) or saline. Animals were sacrificed after $12 \mathrm{hr}$ and liver recovered and stored at $-80^{\circ} \mathrm{C}$ before RNA extraction. Total RNA was prepared from liver using the Trizol reagent (Life Technologies). Twenty $\mu \mathrm{g}$ of total RNA was converted to cDNA with SuperScript reverse transcriptase (Gibco-BRL), using T7-oligo-d(T) 24 as a primer. Second-strand synthesis was performed using T4 DNA polymerase and E. Coli DNA ligase followed by blunt ending by T4 polynucleotide kinase. cDNA was isolated by phenol-chloroform extraction using phase lock gels (Brinkmann). cDNA was in vitro transcribed using the T7 BioArray High Yield RNA Transcript Labeling Kit (Enzo Biochem, New York, N.Y.) to produce biotinylated cRNA. Labelled cRNA was isolated using an RNeasy Mini Kit column (Qiagen). Purified cRNA was fragmented to 200300 mer cRNA using a fragmentation buffer $(100 \mathrm{mM}$ potassium acetate- $30 \mathrm{mM}$ magnesium acetate- $40 \mathrm{mM}$ Tris-acetate, $\mathrm{pH} 8.1$ ), for $35 \mathrm{~min}$ at $94^{\circ} \mathrm{C}$. The quality of total RNA, cDNA synthesis, cRNA amplification and 
cRNA fragmentation was monitored by micro-capillary electrophoresis (Bioanalizer 2100, Agilent Technologies). The cRNA probes were hybridized to an Mu11K oligonucleotide arrays (Affymetrix, Santa Clara, CA). The Mu11K oligonucleotide arrays represents $\sim 6,000$ sequences of mouse Unigene that have been functionally characterized and $\sim 6,000$ sequences ESTs clusters. Each sequence in the chip is represented by 32 probes : 16 "perfect match" (PM) probes that are complementary to the mRNA sequence and 16 "mismatch" (MM) probes that only differ by a single nucleotide at the central base (more detailed information about the Mu11K oligonucleotide arrays can be obtained in the web site http://www.affyme trix.com). Fifteen micrograms of fragmented cRNA was hybridized for $16 \mathrm{~h}$ at $45^{\circ} \mathrm{C}$ with constant rotation $(60$ $\mathrm{rpm})$. Microarrays were processed in an Affymetrix GeneChip Fluidic Station 400. Staining was made with streptavidin-conjugated phycoerythrin (SAPE) followed by amplification with a biotinylated anti-streptavidin antibody and a second round of SAPE, and then scanned using an Agilent GeneArray Scanner (Agilent Technologies). Expression value (signal) is calculated using Affymetrix Genechip software MAS 5.0 (for fully description of the statistical algorithms see http://affymetrix.com/sup port/technical/whitepapers/sadd whitepaper.pdf).

Briefly, signal is calculated as follow : First, probe cell intensities are processed for global background. Then, $\mathrm{MM}$ value is calculated and subtracted to adjust the PM intensity in order to incorporate some measure of nonspecific cross-hybridization to mismatch probes. Then, this value is log-transformed to stabilize the variance. Signal is output as the antilog of the resulting value. The 20 probe pairs representing each gene are consolidated into a single expression level. Finally, software scales the average intensity of all genes on each array within a data set. Final value of signal is considered representative of the amount of transcript in solution.

Housekeeping controls $\beta$-actin and GAPDH genes serve as endogenous controls and are useful for monitoring the quality of the target. Their respective probe sets are designed to be specific to the $5^{\prime}$, middle, or $3^{\prime}$ portion of the transcript. The $3^{\prime} / 5^{\prime}$ signal ratio from these probe sets is informative about the reverse transcription and in vitro transcription steps in the sample preparation. Then, an ideal target in which all transcripts was full-length transcribed would have an identical amount of signal 3' and $5^{\prime}$ and the ratio would be equal to 1 . Differences greater than three fold between signal at $3^{\prime}$ and $5^{\prime}$ for these housekeeping genes indicate that RNA was incompletely transcribed or target may be degraded. Ratio of fluorescent intensities for the 5' and 3' ends of these housekeeping genes was $<2$.
Hybridization experiments were repeated twice using independent cRNA probes synthezised with RNA from two independent livers. Genes were considered as differentially expressed when both hybridizations showed $>2$ folds change. Data presented in this work represent the average of both hybridizations.

\section{RT-PCR analysis}

Total RNA $(1 \mu \mathrm{g})$ was analyzed by RT-PCR with the SuperScript ${ }^{\mathrm{TM}}$ One-step RT-PCR System and the Platinum Taq kit (Life Technologies). RT-PCR was performed using different numbers of cycles to verify that the conditions chosen were within the linear range. The mRNA coding for $\mathrm{p} 8$ was specifically amplified with sense (5'GAAGCTGCTGCCAATACCAACC3') and antisense (5'TAGCTCTGCCCGTCTACCCTC3') primers, in positions 181 and 540 of the cDNA (accession \# AF131196), respectively. The mRNA coding for HMG1 was specifically amplified with sense (5'GGGACTATTAGGATCAAGCAATC3') and antisense (5'CCTAAACTAAGCAGATTAAGG3') primers, in positions 1501 and 1987 of the cDNA (accession \# Z11997), respectively. As control, the transcript coding for the ribosomal protein RL3 was specifically amplified for 22 cycles with sense (5'GAAAGAAGTCGTGGAGGCTG3') and antisense (5'ATCTCATCCTGCCCAAACAC3') primers, in positions 216 and 637 of the cDNA, respectively (accession \# NM_013762).

\section{Competing interests}

None declared.

\section{Authors' contributions}

SV developed p8-/- mice, AH carried out LPS treatments and RNA purification, AG-M and $\mathrm{MB}$ was in charge of microarray hybridization, LS-M carried out some enzymatic dosages, PB participated in the analysis of gene expression data, FF carried out some enzymatic dosages and participated in design of the study, DC carried out RTPCR controls and participated in design of the study, JCD participated in the design of the study, JLI participated in the analysis of data and wrote the manuscript. All authors read and approved the final manuscript.

\section{Additional material}

\section{Additional File 1}

Tables 1 and 2

Click here for file

[http://www.biomedcentral.com/content/supplementary/1471-

230X-3-25-s1.doc]

Additional File 2

Tables 3 and 4

Click here for file 
[http://www.biomedcentral.com/content/supplementary/1471230X-3-25-s2.doc]

\section{Additional File 3}

Tables 5 and 6

Click here for file

[http://www.biomedcentral.com/content/supplementary/1471-

230X-3-25-s3.doc]

\section{Acknowledgements}

We thank to Dr. E. Calvo for access to microarray facilities and interpretation of data. Supported by grants from Société de Secours des Amis des Sciences and EMBO short fellowship (S.V.), Fondation pour la Recherche Médicale (A.H.), poste orange INSERM (A.G.M.). This work was supported by a grant from the Association pour la Recherche sur le Cancer (JLI) and FIS 02027 (DC).

\section{References}

I. Mallo GV, Fiedler F, Calvo EL, Ortiz EM, Vasseur S, Keim V, Morisset $\mathrm{J}$ and lovanna JL: Cloning and expression of the rat p8 cDNA, a new gene activated in pancreas during the acute phase of pancreatitis, pancreatic development, and regeneration, and which promotes cellular growth. J Biol Chem 1997, 272:32360-32369.

2. Vasseur S, Mallo GV, Fiedler F, Bodeker H, Canepa E, Moreno S and lovanna JL: Cloning and expression of the human p8, a nuclear protein with mitogenic activity. Eur J Biochem 1999, 259:670-675.

3. Encinar JA, Mallo GV, Mizyrycki C, Giono L, Gonzalez-Ros JM, Rico $M$, Canepa E, Moreno S, Neira JL and lovanna JL: Human p8 is a HMG-I/Y-like protein with DNA binding activity enhanced by phosphorylation. J Biol Chem 200I, 276:2742-275I.

4. Piekielko A, Drung A, Rogalla P, Schwanbeck R, Heyduk T, Gerharz $M$, Bullerdiek J and Wisniewski JR: Distinct organization of DNA complexes of various $\mathrm{HMGI} / \mathrm{Y}$ family proteins and their modulation upon mitotic phosphorylation. J Biol Chem 200I, 276: 1984-1992.

5. Andersson $\mathrm{U}$, Erlandsson-Harris $\mathrm{H}$, Yang $\mathrm{H}$ and Tracey KJ: HMGB as a DNA-binding cytokine. J Leukoc Biol 2002, 72: 1084-I09I.

6. Jiang YF, Vaccaro MI, Fiedler F, Calvo EL and lovanna JL: Lipopolysaccharides induce p8 $\mathrm{mRNA}$ expression in vivo and in vitro. Biochem Biophys Res Commun 1999, 260:686-690.

7. Ree AH, Tvermyr M, Engebraaten O, Rooman M, Rosok O, Hovig E, Meza-Zepeda LA, Bruland OS and Fodstad O: Expression of a novel factor in human breast cancer cells with metastatic potential. Cancer Res 1999, 59:4675-4680.

8. Vasseur S, Hoffmeister A, Garcia S, Bagnis C, Dagorn JC and lovanna $\mathrm{JL}: \mathbf{p 8}$ is critical for tumour development induced by rasV $\mathrm{I} 2$ mutated protein and EIA oncogene. EMBO Rep 2002, 3:165-170.

9. Bratland A, Risberg K, Maelandsmo GM, Gutzkow KB, Olsen OE, Moghaddam A, Wang MY, Hansen CM, Blomhoff HK, Berg JP, Fodstad $\mathrm{O}$ and Ree $\mathrm{AH}$ : Expression of a novel factor, com I, is regulated by I,25-dihydroxyvitamin D3 in breast cancer cells. Cancer Res 2000, 60:5578-5583.

10. Macdonald J, Galley HF and Webster NR: Oxidative stress and gene expression in sepsis. Br J Anaesth 2003, 90:22I-232.

II. Spolarics Z, Stein DS and Garcia ZC: Endotoxin stimulates hydrogen peroxide detoxifying activity in rat hepatic endothelial cells. Hepatology 1996, 24:691-696.

12. Sass G, Heinlein S, Agli A, Bang R, Schumann J and Tiegs G: Cytokine expression in three mouse models of experimental hepatitis. Cytokine 2002, 19:115-120.

13. Calogero S, Grassi F, Aguzzi A, Voigtlander T, Ferrier P, Ferrari S and Bianchi ME: The lack of chromosomal protein Hmg I does not disrupt cell growth but causes lethal hypoglycaemia in newborn mice. Nat Genet 1999, 22:276-280.
14. Grosschedl R, Giese K and Pagel J: HMG domain proteins: architectural elements in the assembly of nucleoprotein structures. Trends Genet 1994, 10:94-100.

15. Reeves R and Nissen MS: Cell cycle regulation and functions of HMG-I(Y). Prog Cell Cycle Res I995, I:339-349.

16. Bewley CA, Gronenborn AM and Clore GM: Minor groove-binding architectural proteins: structure, function, and DNA recognition. Annu Rev Biophys Biomol Struct 1998, 27:105-131.

17. Tallini $G$ and $\mathrm{Dal} \mathrm{Cin} P$ : HMGI(Y) and HMGI-C dysregulation: a common occurrence in human tumors. Adv Anat Pathol 1999, 6:237-246.

18. Hamilton TA, Ohmori Y, Tebo JM and Kishore R: Regulation of macrophage gene expression by pro- and anti-inflammatory cytokines. Pathobiology 1999, 67:24|-244.

19. Forstermann $\mathrm{U}$, Boissel JP and Kleinert $\mathrm{H}$ : Expressional control of the 'constitutive' isoforms of nitric oxide synthase (NOS I and NOS III). FASEB J 1998, I 2:773-790.

20. Hashimoto SI, Suzuki T, Nagai S, Yamashita T, Toyoda N and Matsushima K: Identification of genes specifically expressed in human activated and mature dendritic cells through serial analysis of gene expression. Blood 2000, 96:2206-22I4.

21. Ichikawa JK, Norris A, Bangera MG, Geiss GK, van't Wout AB, Bumgarner RE and Lory S: Interaction of pseudomonas aeruginosa with epithelial cells: identification of differentially regulated genes by expression microarray analysis of human CDNAs. Proc Natl Acad Sci USA 2000, 97:9659-9664.

22. Rosenberger CM, Scott MG, Gold MR, Hancock RE and Finlay BB: Salmonella typhimurium infection and lipopolysaccharide stimulation induce similar changes in macrophage gene expression. J Immunol 2000, 164:5894-5904.

23. Smith JB and Herschman HR: The glucocorticoid attenuated response genes GARG-16, GARG-39, and GARG-49/IRG2 encode inducible proteins containing multiple tetratricopeptide repeat domains. Arch Biochem Biophys 1996, 330:290-300.

24. Liao F, Lusis AJ, Berliner JA, Fogelman AM, Kindy M, de Beer MC and de Beer FC: Serum amyloid A protein family. Differential induction by oxidized lipids in mouse strains. Arterioscler Thromb 1994, 14:1475-1479.

25. Macarthur H, Westfall TC, Riley DP, Misko TP and Salvemini D: Inactivation of catecholamines by superoxide gives new insights on the pathogenesis of septic shock. Proc Natl Acad Sci USA 2000, 97:9753-9758.

26. Hoffmeister A, Ropolo A, Vasseur S, Mallo GV, Bodeker H, Ritz-Laser B, Dressler GR, Vaccaro MI, Dagorn JC, Moreno S and lovanna JL: The HMG-I/Y-related protein p8 binds to p300 and Pax2 trans-activation domain-interacting protein to regulate the trans-activation activity of the Pax2A and Pax2B transcription factors on the glucagon gene promoter. J Biol Chem 2002, 277:223।4-223।9.

27. Kuhbandner S, Brummer S, Metzger D, Chambon P, Hofmann F and Feil $R$ : Temporally controlled somatic mutagenesis in smooth muscle. Genesis 2000, 28:15-22.

28. Vasseur S, Hoffmeister A, Garcia-Montero A, Mallo GV, Feil R, Kuhbandner S, Dagorn JC and lovanna JL: p8-deficient fibroblasts grow more rapidly and are more resistant to adriamycininduced apoptosis. Oncogene 2002, 21:1685-1694.

29. de ca J, Cuadrado S, Vallet J, Benasco C, Martin E, Ardanuy C, Closa $D$, Hotter G and Jaurrieta E: Protective effects of lazaroid U74389G on intestinal graft after heterotopic small bowel transplantation in rats. J Surg Res 1998, 75: 18-23.

\section{Pre-publication history}

The pre-publication history for this paper can be accessed here:

\section{http://www.biomedcentral.com/1471-230X/3/25/pre} pub 